\title{
Simulation of a dc electron beam strongly influenced by self-fields
}

\author{
G. H. Zapalac \\ Imatron Inc., 389 Oyster Point Boulevard, South San Francisco, California 94080
}

(Received 22 April 1999; published 15 November 1999)

\begin{abstract}
A method is presented to simulate the transport of a dc charged particle beam strongly influenced by its self-fields. If the initial phase space of the beam is chosen to be a Kapchinskij-Vladimirskij distribution, the simulation is in agreement with the Kapchinskij-Vladimirskij envelope equations. The simulation was initialized with a Gaussian transverse momentum distribution to investigate the origin of current profiles measured in a $130 \mathrm{keV}, 635 \mathrm{~mA}$ plasma-focused electron beam.
\end{abstract}

PACS numbers: 41.75.Fr, 07.05.Tp, 41.85.Ew, 41.85.Ja

\section{INTRODUCTION}

Beam self-forces influence the behavior of plasmafocused electron beams, and also the behavior of any charged particle beam that does not satisfy the condition $\beta=v / c \approx 1$. These forces include both space charge repulsion and focusing due to the self-magnetic field. This report describes a method to simulate a dc beam with a behavior that may be dominated by the beam self-fields. The simulation randomly populates an initial phase space distribution with particles. These particles are propagated in small steps through the transport region using the combined self- and external fields. At the beginning of each step, the particles are binned on a rectangular grid to determine the current density; the current density is then used to compute the self-fields for that step.

The simulation was designed for distributed processing using a single server with multiple clients. Each client generates a fraction of the total number of particles. These particles are binned on a grid to determine the current profile, and the gridded profile is sent to the server. The server accumulates the profiles from all of the clients for the purpose of combining the statistics from all of the particles. The accumulated profile is then sent back to each of the clients. Each client computes the self-fields using the accumulated profile, adds the external fields, and propagates its particles through a small step using the combined self- and external fields. The particles are next rebinned to determine the new current profile, and the sequence is repeated.

The simulation was used to model the transport of a $130 \mathrm{keV}(\beta=0.604), 635 \mathrm{~mA}$ electron beam in the presence of a rarefied background gas. This beam is used to generate $\mathrm{x}$ rays in a computed tomography scanner used for medical imaging. Ions created by the beam in the background gas neutralize the beam if they are not removed. The ratio of ion to electron charge densities will be denoted as the beam neutralization fraction $f$. The condition $f=1$ over the entire beam profile represents a completely neutralized beam influenced only by its self-magnetic field. The condition $f=0$ over the entire profile represents an unneutralized beam influenced by both its self-magnetic and self-electric fields. The $f=0$ condition may be created by applying a transverse electric field to sweep ions out of the beam more rapidly than they are generated [1]. The simulation was performed assuming an abrupt transition between an initial $f=0$ region and a final $f=1$ region.

The initial transverse momenta $p_{x}$ and $p_{y}$ of the electron beam were assumed to have a Gaussian distribution with a spread determined by the temperature of the cathode in the electron gun; the term "Maxwellian" will be used to refer to such a beam. The author is not aware of an analytical approach to predict the detailed behavior of a Maxwellian beam influenced by self-forces. However, if the initial phase space of the beam is contrived to follow a Kapchinskij-Vladimirskij (KV) distribution, the beam profile is always uniform and elliptical with linear self-forces, and its evolution is given by the $\mathrm{KV}$ envelope equations. Hence the KV beam provides a theoretical check of the simulation. Results are therefore presented for both Maxwellian and KV simulations of plasma-focused electron beams.

The KV distribution constrains all of the particles to an ellipsoidal shell in phase space [2],

$g\left(x, y, x^{\prime}, y^{\prime}\right)=\delta\left(\frac{x^{2}}{X^{2}}+\frac{y^{2}}{Y^{2}}+\frac{X^{2} x^{\prime 2}}{\epsilon_{x}^{2}}+\frac{Y^{2} y^{\prime 2}}{\epsilon_{y}^{2}}-1\right)$,

where $\epsilon_{x}$ and $\epsilon_{y}$ denote the emittances in the $x$ - $p_{x}$ and $y-p_{y}$ planes, and $X$ and $Y$ are the semiaxes of the beam profile. The approximations $x^{\prime}=d x / d s \approx$ $\hat{p}_{x}$ and $y^{\prime}=d y / d s \approx \hat{p}_{y}$ are used, where $s$ denotes the position along the reference trajectory. The KV distribution yields uniform elliptical profiles in all twodimensional projections of phase space. The KV envelope equations describe the evolution of the semiaxes of the profile ellipse as functions of position $s$. In the absence of external fields, these equations are [2]

$$
\begin{aligned}
& \frac{d^{2} X(s)}{d s^{2}}-\frac{2 K}{X+Y}-\frac{\epsilon_{x}^{2}}{X^{3}}=0, \\
& \frac{d^{2} Y(s)}{d s^{2}}-\frac{2 K}{X+Y}-\frac{\epsilon_{y}^{2}}{Y^{3}}=0,
\end{aligned}
$$


and the perveance $K$ is given by

$$
K=\frac{2 N_{e} r_{e}}{\beta^{2} \gamma}\left(1-f-\beta^{2}\right),
$$

where $r_{e}$ is the classical electron radius and $N_{e}$ is the number of particles per unit length of the beam. These equations may be integrated in the presence of transverse fields.

The KV equations are not valid in the presence of longitudinal fields [2]. Significant longitudinal magnetic fields are present in the experimental transport line, so the $\mathrm{KV}$ beam is simulated for a hypothetical transport line where only transverse external fields are present.

\section{EVOLUTION OF THE CURRENT DENSITY}

\section{A. Self-fields}

The self-fields are determined by computing the electrostatic potential $\phi(x, y)$ from the current density $J(x, y)$. The variation of $J$ in the direction of the beam is assumed gradual enough to be neglected, and the influence of conducting surfaces such as the beam pipe and ion-clearing electrodes is also neglected. The potential is differentiated numerically to obtain the self-fields,

$$
\begin{aligned}
& \mathbf{E}_{s}(x, y)=-\nabla \phi(x, y), \\
& \mathbf{B}_{s}(x, y)=\frac{\vec{\beta}}{c} \times \mathbf{E}_{s}(x, y) .
\end{aligned}
$$

The current density is determined by binning the electrons on a rectangular grid with an equal number of elements along the $x$ and $y$ directions. The dimensions $L_{x}$ and $L_{y}$ of the grid are adjusted to match the aspect ratio of the beam profile. Two different fast Fourier transform (FFT) based algorithms were used to compute the potential from the current density: Hockney's algorithm [3] and an independently developed algorithm denoted as the elliptical image algorithm. The remainder of the discussion in this section describes Hockney's algorithm, which is faster and more easily implemented; the elliptical image algorithm is detailed in Appendix A.

Hockney's algorithm computes the potential using the convolution of the current density

$$
\phi(x, y)=\int J\left(x^{\prime}, y^{\prime}\right) G\left(x-x^{\prime}, y-y^{\prime}\right) d x^{\prime} d y^{\prime},
$$

where the convolution kernel due to a filament of current at the origin is given by

$$
G(x, y)=-\frac{\mu_{0} c}{2 \pi \beta} \log \sqrt{x^{2}+y^{2}} .
$$

The value of the kernel at the origin is set to zero (Appendix B).

The convolution is performed using a two-dimensional FFT. The Fourier domain of the kernel covers the region $0 \leq x<2 L_{x}, 0 \leq y<2 L_{y}$. The kernel is implemented in wrap-around fashion, reflected across the horizontal and vertical lines that divide the Fourier domain into quadrants. Because the kernel extends beyond the lower left quadrant of the Fourier domain, there are cusps along the horizontal and vertical lines bisecting the Fourier domain. When the Fourier domain is periodically repeated, the kernel depicts the physically correct potential from an isolated current filament at the origin only within the restricted region $-L_{x}<x<L_{x},-L_{y}<y<L_{y}$.

Hockney's algorithm restricts the beam profile to the lower left quadrant of the Fourier domain containing the origin; the remainder of the grid is padded with zeros. The convolution procedure of Eq. (2.2) always uses the physically correct region of the kernel within the quadrant containing the beam profile.

\section{B. External fields and particle propagation}

The external fields were calculated in the laboratory frame using boundary element models for the magnets in the transport line ${ }^{1}$; the electric fields from the ion-clearing electrodes were neglected. Unlike the self-fields, the external fields vary slowly across the profile of the beam. This allows a derivative expansion of the external fields about the reference trajectory. If we refer to the point on the reference trajectory as $\mathbf{r}_{R}$ and use the subscript $k$ to refer to the $k$ th component of the external field, we may write to first order

$$
F_{k}\left(\mathbf{r}_{R}+d \mathbf{r}\right)=F_{k}\left(\mathbf{r}_{R}\right)+\nabla F_{k} \cdot d \mathbf{r},
$$

where $\mathbf{r}_{R}+d \mathbf{r}$ is the electron position and $F$ refers to either the magnetic or (if present) the electric external field. If sextupole components of the external fields are important, Eq. (2.4) must be expanded to second order. Sextupole fields are neglected in the remainder of this discussion.

The nine magnetic field derivatives required in Eq. (2.4) were determined by using the boundary element model to calculate the fields at $\mathbf{r}_{R}$ and six nearby positions: $\mathbf{r}_{R} \pm \hat{x} \delta x, \mathbf{r}_{R} \pm \hat{y} \delta y$, and $\mathbf{r}_{R} \pm \hat{z} \delta z$. Derivatives were constructed by taking differences between the fields. After the derivatives were calculated, they were corrected to force agreement with Maxwell's equations. This correction helps to reduce small errors introduced by the calculation. The curl relations $\nabla \times \mathbf{B}=0$ require $\partial B_{k} / \partial r_{j}=\partial B_{j} / \partial r_{k}$. To ensure compliance with the curl, the derivatives on each side of the equation were averaged. Both derivatives were then replaced with this average. Because of numerical error, the sum of the derivatives $\partial B_{k} / \partial r_{k}$ calculated for the divergence $\nabla \cdot \mathbf{B}$ is not identically zero, but equal to a small number $\Delta_{B}$. These derivatives were corrected by the following

\footnotetext{
${ }^{1}$ Amperes 3D Magnetostatic Design Software Version 2.1 by Integrated Engineering Software.
} 
prescription:

$$
\frac{\partial B_{k}}{\partial r_{k}} \rightarrow \frac{\partial B_{k}}{\partial r_{k}}-\frac{\Delta_{B} B_{k}^{2}\left(\mathbf{r}_{R}\right)}{\left|\mathbf{B}\left(\mathbf{r}_{R}\right)\right|^{2}}
$$

The electrons were propagated in small steps in the laboratory frame under the combined influence of the self- and external fields using the leapfrog algorithm [3]. The step length $\delta s$ was chosen to be proportional to the smallest rms width of the beam in either $x$ or $y$. For the simulations presented here, the proportionality constant used was $\kappa=\frac{1}{2}$. The variable step length scales with the self-fields, which are proportional to the size of the beam.

\section{Errors}

Errors in the simulation include errors in calculating the self-fields, errors in calculating the external fields, and errors introduced from the finite step size. In the previous section we have already discussed steps that were taken to reduce errors from introducing the external fields. The sensitivity of the simulation to the remaining errors was investigated by choosing a finer grid for the beam profile, decreasing the number of particles in the simulation, and decreasing the step size.

Errors in calculating the self-fields include aliasing during the FFT, the error introduced by the numerical derivative to obtain the electric field from the electrostatic potential, and statistical fluctuations when determining the current profile from binning the particles.

The KV beam is potentially sensitive to all of the errors listed above because the beam profile ends abruptly at the profile boundary throughout the transport line. The KV beam covered about $100 \times 100$ grid elements, and the entire Fourier domain used $256 \times 256$ elements. At this grid density statistical errors dominated the error for runs with less than several million electrons. Errors introduced by insufficient statistics degrade the uniformity and elliptical symmetry in one or more of the phase space projections.

The simulation of the Maxwellian beam used the same number of grid elements as the KV beam; it was found to be much more stable for reduced statistics.

\section{KV AND MAXWELLIAN BEAM SIMULATIONS}

\section{A. Generation of the initial phase space}

The initial current density for examples presented in this report was a round uniform or nearly uniform profile

$$
J_{0}(\eta=r / a)=\frac{I_{0}}{N(a, \Lambda)\left[1+\exp \left(\Lambda^{2}\left(\eta^{2}-1\right)\right)\right]},
$$

where $I_{0}$ is the total current of the beam and $N(a, \Lambda)$ is a normalization constant

$$
N(a, \Lambda)=\frac{\pi a^{2} \log \left[1+\exp \left(\Lambda^{2}\right)\right]}{\Lambda^{2}} .
$$

The parameters $a$ and $\Lambda$ control the radial extent and rate of cutoff of the profile. The normalization condition is given by

$$
2 \pi a^{2} \int_{0}^{\infty} J_{0}(\eta) \eta d \eta=I_{0}
$$

As $\Lambda$ increases, the edge of the beam becomes sharper; the value $\Lambda=10$ generates an essentially uniform profile with radius $a$ suitable for simulating a KV distribution. The initial profile for the Maxwellian beam was generated with smaller values of $\Lambda$ so that the current density at the edge of the profile decreased more gradually. Figure 1 shows a slice through the center of the profile used for the simulation reported here with $\Lambda=2$.

Three random numbers $u_{1}, u_{2}$, and $u_{3}$ between 0 and 1 were used to generate the initial positions and transverse momenta for the $\mathrm{KV}$ beam. Although there are four variables $x, y, p_{x}$, and $p_{y}$, these are constrained by the phase space ellipsoid of Eq. (1.1). Equation (3.2) was used to map $u_{1}$ into the unit area underneath the normalized beam profile distribution in order to solve numerically for the integration limit $\eta_{u}$,

$$
u_{1}=\frac{2 \pi a^{2}}{I_{0}} \int_{0}^{\eta_{u}} J(\eta) \eta d \eta .
$$

The numbers $u_{2}$ and $u_{3}$ provide angles $\theta=2 \pi u_{2}$ and $\phi=2 \pi u_{3}$ in the $x-y$ and $p_{x}-p_{y}$ planes. The phase space variables are given by

$$
\begin{aligned}
x & =a \eta_{u} \cos \theta, \\
y & =a \eta_{u} \sin \theta, \\
x^{\prime} & =\epsilon \sqrt{1-\eta_{u}^{2}} \cos \phi / a, \\
y^{\prime} & =\epsilon \sqrt{1-\eta_{u}^{2}} \sin \phi / a .
\end{aligned}
$$

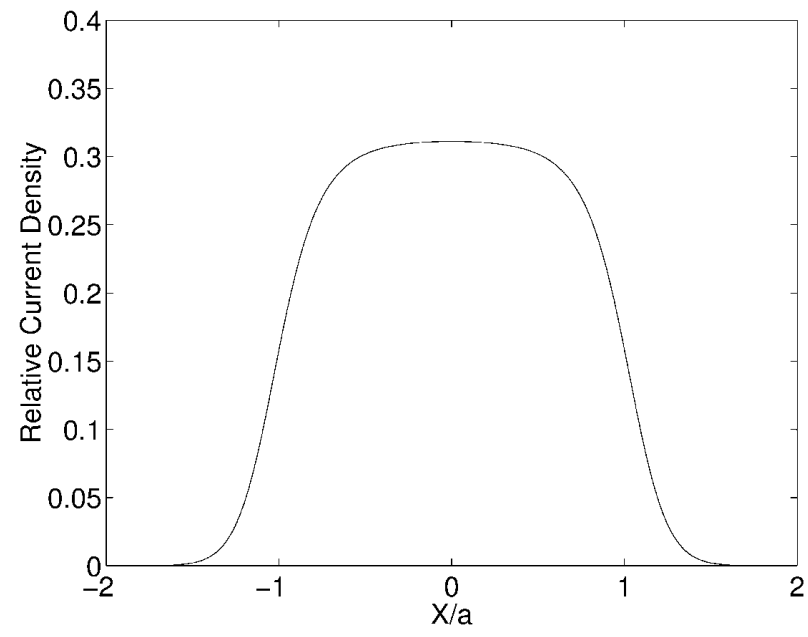

FIG. 1. The beam profile distribution function [Eq. (3.1)] plotted at $y=0$ using $\Lambda=2$. 
For the case of the round Maxwellian profile, the emittance was also used to determine the transverse momentum distribution, but four random numbers are required because $p_{x}$ and $p_{y}$ are uncorrelated. The rms emittance $\overline{\boldsymbol{\epsilon}}$ in the $x-p_{x}$ plane is given by [2]

$$
\bar{\epsilon}=4 \sqrt{\left\langle x^{2}\right\rangle\left\langle x^{\prime 2}\right\rangle-\left\langle x x^{\prime}\right\rangle^{2}}
$$

with a similar expression for the $y-p_{y}$ plane. The quantity $\left\langle x x^{\prime}\right\rangle$ is zero because the phase space variables are now uncorrelated. The variables $x^{\prime}$ and $y^{\prime}$ were each chosen at random from a Gaussian distribution with a standard deviation determined by the temperature $T$ of the cathode [2]

$$
\sigma^{2}=k T /\left(m_{e} \beta^{2} c^{2}\right)=\left\langle x^{\prime 2}\right\rangle=\left\langle y^{\prime 2}\right\rangle .
$$

For a round uniform beam of radius $a,\left\langle x^{2}\right\rangle=a^{2} / 4$ and $\bar{\epsilon}=2 a \sigma$. In general, $\left\langle x^{2}\right\rangle$ is determined by numerical integration; $\bar{\epsilon}=2.19 a \sigma$ for the distribution shown in Fig. 1. The examples that follow were simulated with the emittance calculated for the experimental transport line: $5.06 \times 10^{-3} \mathrm{~mm} \mathrm{rad}$.

\section{B. Hypothetical transport line with a KV beam}

A KV beam with an initially round current profile of radius $3.2 \mathrm{~mm}$ was simulated in a $120 \mathrm{~cm}$ hypothetical transport line. The first $10 \mathrm{~cm}$ of the transport line is simulated with $f=0$; the remainder of the transport line is at $f=1$. A quadrupole magnet provides a uniform gradient of $0.5 \mathrm{G} / \mathrm{cm}$ in the region $20<s<40 \mathrm{~cm}$. The focusing due to the quadrupole and self-magnetic field causes the KV beam to pass sequentially through two elliptical waists downstream of the quadrupole at 75 and $91 \mathrm{~cm}$. The major axis of the beam profile is aligned with the $x$ axis at the first waist; at the second waist it is aligned with the $y$ axis.

Figure 2 shows the rms size of the beam in $x$ and $y$ for both the simulation and the KV envelope equations. This particular simulation used $4.5 \times 10^{6}$ electrons in order to obtain uniform elliptical phase space plots at and beyond the second waist, but equally good agreement with the envelope equations is obtained with far fewer electrons (under 50 000).

\section{Experimental transport line with a Maxwellian beam}

The experimental transport line simulation also has an initial section where $f=0$ and a quadrupole to separate the waists in the $x-s$ and $y-s$ planes; however, it is $260 \mathrm{~cm}$ in length and contains a solenoid and dipole deflection magnet. The dipole coils surround the quadrupole coils and deflect the beam $30^{\circ}$ in the $y-z$ plane. The solenoid magnet is positioned upstream of the quadrupole and dipole within the $f=0$ region. The $f=0$ region extends $49 \mathrm{~cm}$ beyond the initial waist of the beam where the simulation is started.
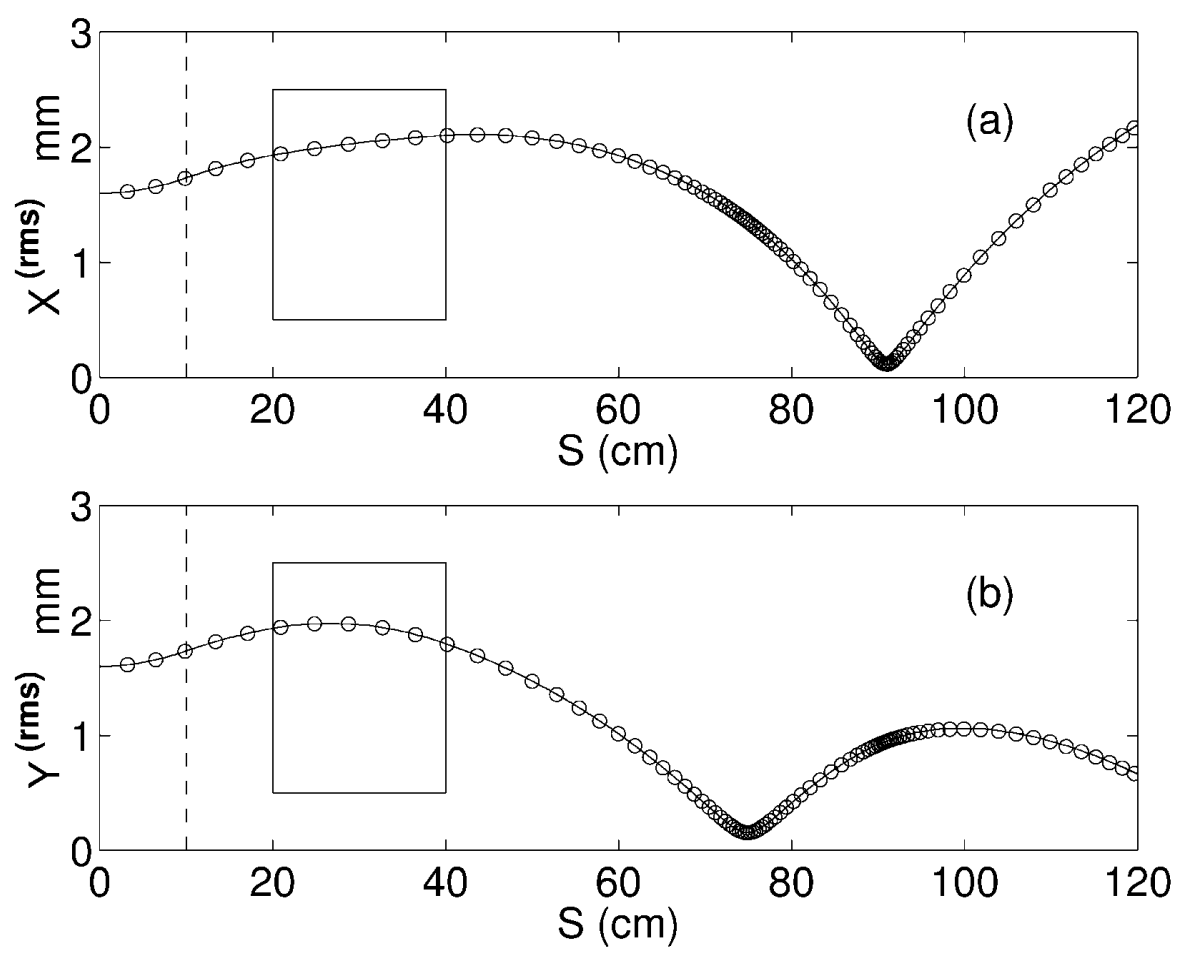

FIG. 2. The rms half-width of the profile predicted by the simulation for a KV electron beam in a hypothetical transport line for (a) the $x$-s plane and (b) the $y$-s plane. The KV equations are used to generate the solid line; open circles are from the simulation. The box marks the relative $s$ position of the quadrupole magnet. The transition from $f=0$ to $f=1$ is shown by the vertical dashed line. All figures are from simulations using Hockney's algorithm unless otherwise noted. 
The partially neutralized transition region between $f=$ 0 and $f=1$ was controlled by applying voltage to an ion trap. This transition region is about $4 \mathrm{~cm}$ in length and has been simulated in a separate ion simulation, which generates and propagates ions in small time steps until equilibrium is reached under the combined influence of the electric fields from the ions, electrodes, and electron beam. Details of the ion simulation are beyond the scope of this report, and the ion simulation was not used for the results presented here, which were simulated assuming a sudden transition between the $f=0$ and $f=1$ regions. The simulation was repeated using the transverse electric fields provided by the ion simulation; it was found that the partially neutralized region enhanced the focusing in both planes, with negligible changes to the beam spot distributions near the final waist.

The beam profile near the final waist in the $x$-s plane is measured experimentally by sweeping the beam spot past a $0.75 \mathrm{~mm}$ diameter wire using the dipole magnet and sampling the current from the wire as a function of time [4]. The profile of the beam on either side of the waist may be observed on the sampling wire by adjusting the current of the solenoid magnet to move the position of the waist.

Figure 3 shows the rms beam envelope from the simulation of a $130 \mathrm{keV}, 635 \mathrm{~mA}$ Maxwellian electron beam. The beam was generated with $1.5 \times 10^{6}$ electrons using the distribution from Eq. (3.1) with $a=1.7 \mathrm{~mm}$ and $\Lambda=2$.
Scatter plots of the beam profile near the final waist in the $x$-s plane are shown in Fig. 4; these plots are at $s_{w}$, $s=s_{w} \pm 8.9 \mathrm{~cm}$, and $s=s_{w} \pm 25.7 \mathrm{~cm}$, where $s_{w}$ is the position of the rms waist. Figure 5 shows a simulation of the sampling wire distributions for these five profiles, and Fig. 6 shows the experimental sampling wire measurements.

The simulation demonstrates that the separation of the Maxwellian beam into two peaks near the final waist is a consequence of the gradual decrease of the current density at the edge of the initial beam profile. The simulated profile does not display two peaks upstream of the final waist if the initial profile is uniform $(\Lambda=10)$. As $\Lambda$ is decreased, the two peaks become more prominent, although the general pattern of behavior shown in Fig. 5 remains the same. The evolution of the current profile into two peaks may be understood from the evolution of the phase space projection $p_{x}$ versus $x$. As the beam expands due to space charge repulsion in the initial $f=$ 0 region, the self-forces on the inner "core" electrons increase linearly with radius because the beam is uniform in this region. The self-forces on the outer electrons in the nonuniform boundary region increase less rapidly with radius, and the outer electrons develop a larger spread in transverse momentum than the core electrons. This increased momentum spread increases the slope $\left|d p_{x} / d x\right|$ of the outer electrons after the beam enters the $f=1$ region, where the transverse momentum reverses sign
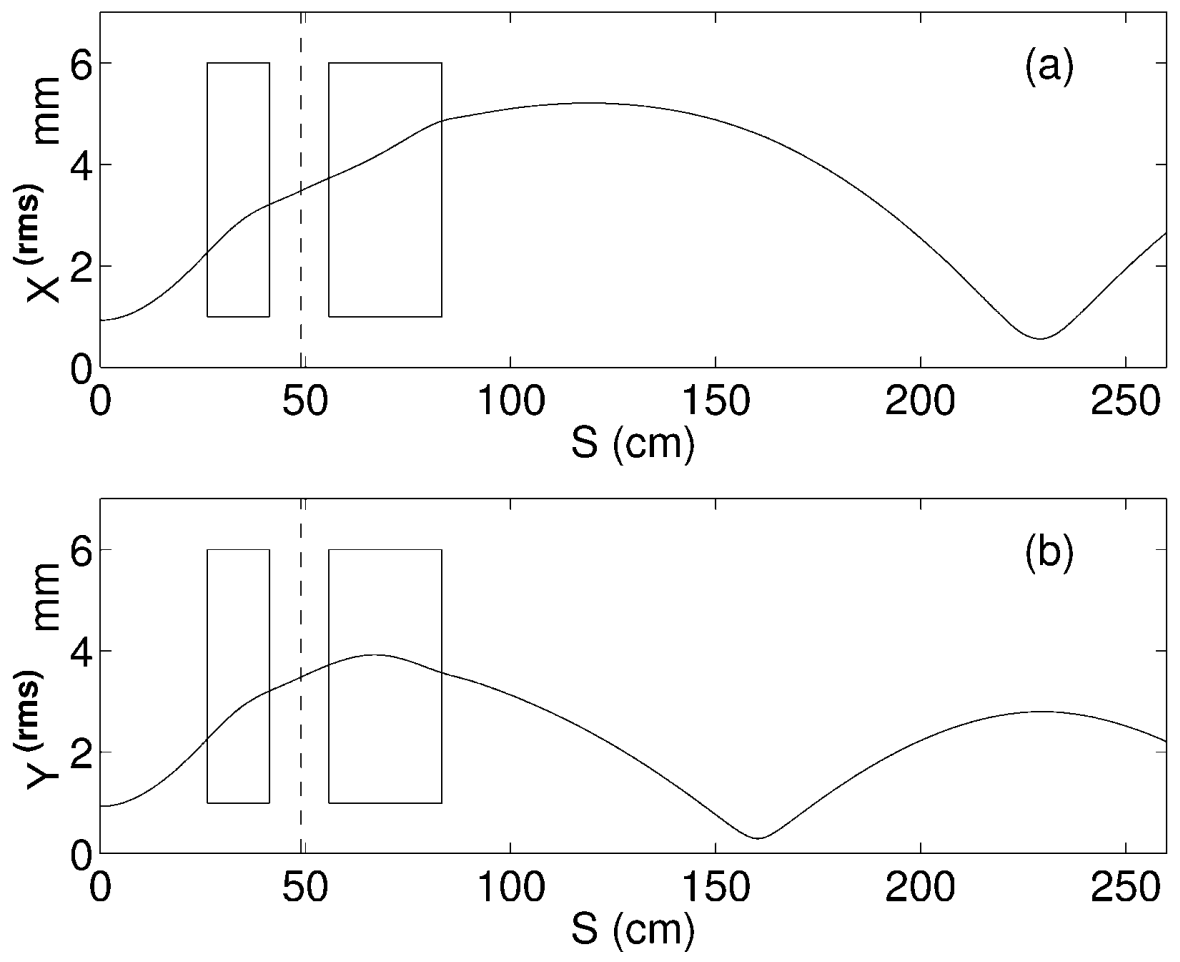

FIG. 3. The rms half-width of the profile predicted by the simulation for a Maxwellian electron beam in the experimental transport line for (a) the $x-s$ plane and (b) the $y$-s plane. The transition from $f=0$ to $f=1$ is shown by the vertical dashed line. Boxes mark the relative $s$ positions of the solenoid and dipole/quadrupole magnets. 

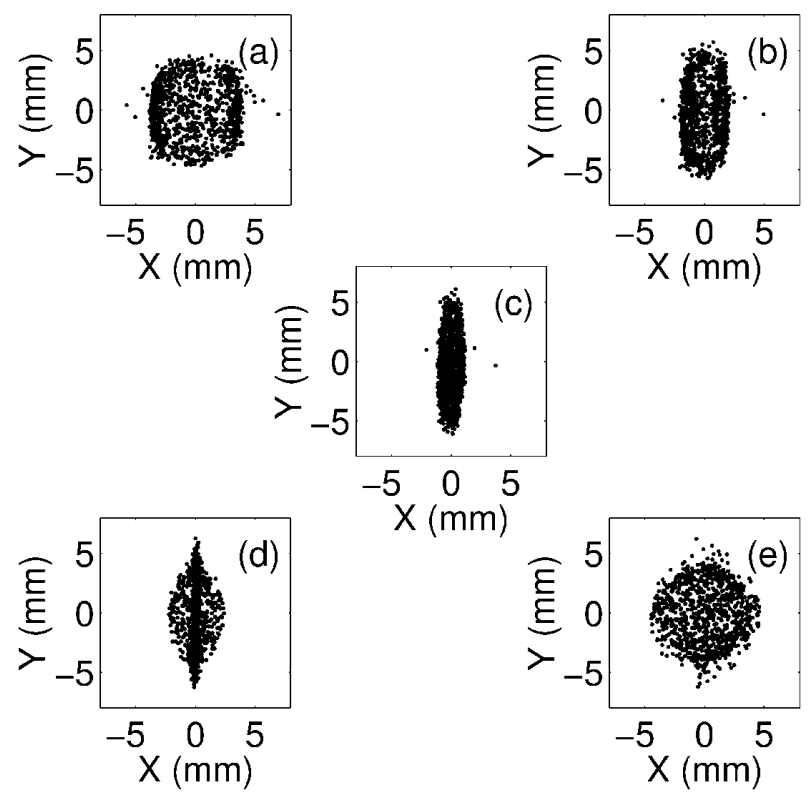

FIG. 4. Scatter plots of electron positions from the experimental transport line simulation near the final waist in the $x-s$ plane at the positions (a) $25.7 \mathrm{~cm}$ upstream of the waist, (b) $8.9 \mathrm{~cm}$ upstream of the waist, (c) at the waist, (d) $8.9 \mathrm{~cm}$ downstream of the waist, and (e) $25.7 \mathrm{~cm}$ downstream of the waist.

due to the attractive force from the self-magnetic field. Figure 7 shows scatter plots of $p_{x}$ versus $x$ at four positions earlier in the transport line, illustrating the evolution of the beam into the distribution shown at $136 \mathrm{~cm}$, where the beam has begun to contract along $x$ as it propagates towards the waist in the $x$-s plane. At this position the
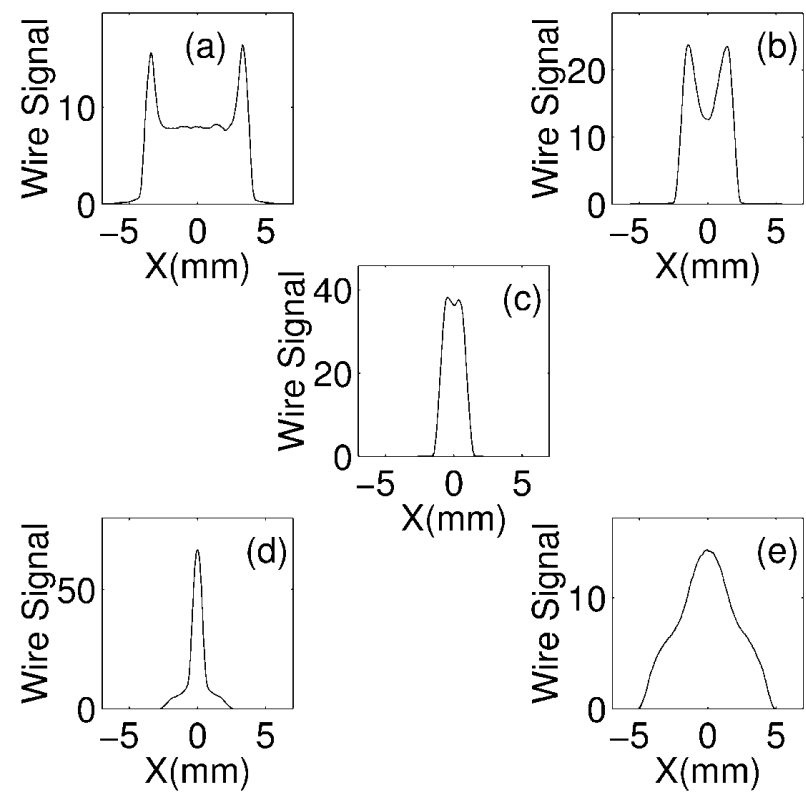

FIG. 5. Simulation of sampling wire distributions for the beam profiles shown in Fig. 4. Relative wire signals are shown on the vertical axes for this figure and for Figs. 6 and 9.

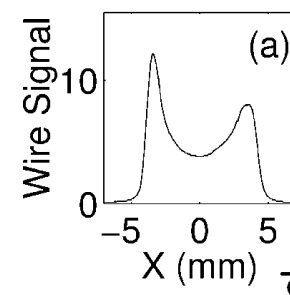

(a)
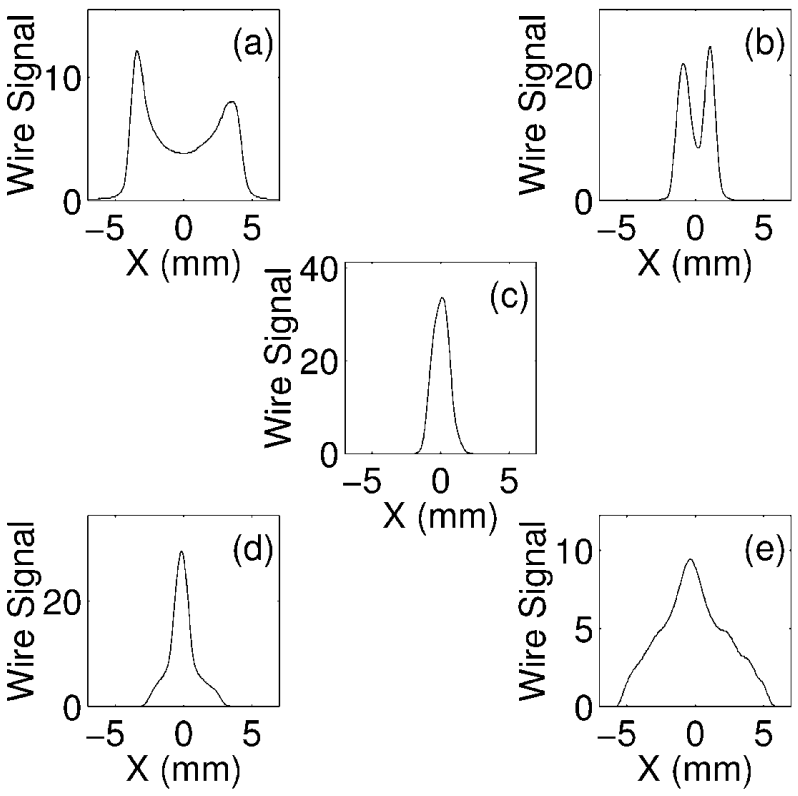

FIG. 6. Experimental sampling wire measurements at five values of the solenoid current, adjusted to sample the profile at the $s$ positions in Fig. 4 .

outer electrons have higher transverse momenta and a greater slope $\left|d p_{x} / d x\right|$ than the core electrons.

Figure 8 shows scatter plots of $p_{x}$ versus $x$ for the five profile distributions in Fig. 4. The core and outer electrons are plotted with different symbols so that their progress may be traced. As the electrons approach the waist in the $x$-s plane, the high-momentum outer electrons move horizontally across the plot towards $x=0$. These electrons overtake the electrons in the core, creating an accumulation of electrons at large $|x|$. At this point the beam profile $x$ distribution assumes a double horned appearance, with peaks at the edges and a wide trough in the middle. As the high-momentum electrons continue to move horizontally across the phase space plot, the double peaks get closer, and the distribution appears as a pair of overlapping peaks. The high-momentum electrons finally cross through a waist in the vicinity of the rms waist of the beam. At the waist of the core electrons the distribution appears as a single peak on top of a localized halo. The core electrons comprise most of the peak, while the highmomentum electrons comprise the halo. Finally, the core electrons begin to expand outward from their waist and the distribution assumes a more triangular shape.

\section{SUMMARY}

This report has described a method to simulate a nonlaminar dc beam which may be strongly influenced by beam self-forces. The simulation propagates a large number of particles under the combined influence of the self- and external fields. The self-fields are calculated by numerical differentiation of the electrostatic potential; 

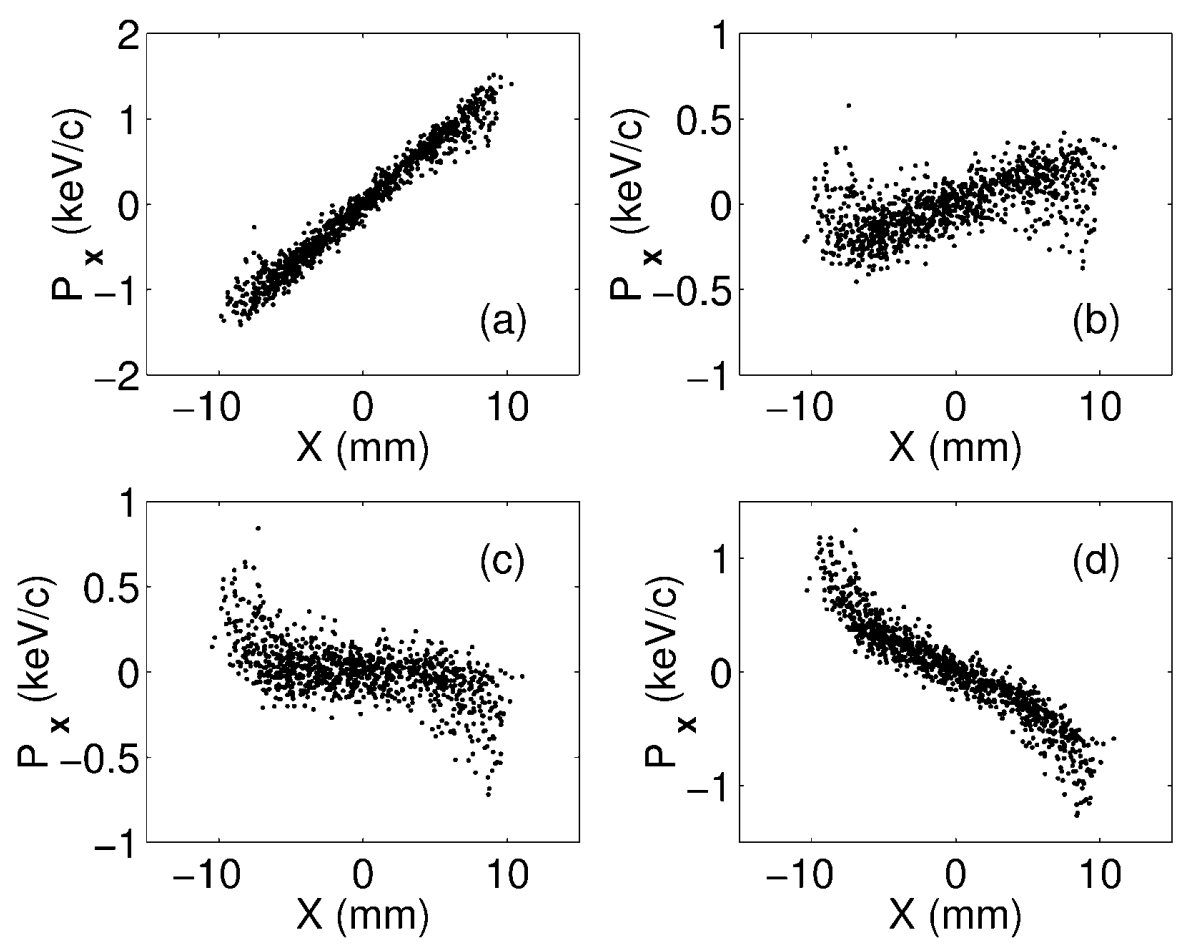

FIG. 7. Scatter plot of the phase space projection $p_{x}$ versus $x$ from the experimental transport line simulation at (a) $s=85$, (b) 116 , (c) 124 , and (d) $136 \mathrm{~cm}$. At $136 \mathrm{~cm}$, electrons at large $|x|$ have higher transverse momenta and a greater slope $\left|d p_{x} / d x\right|$ than the inner core electrons.

Hockney's algorithm is used to determine the potential by convolution of the current density with the potential from a filament of current. The simulation is well suited for distributed processing; it has been used on a small number
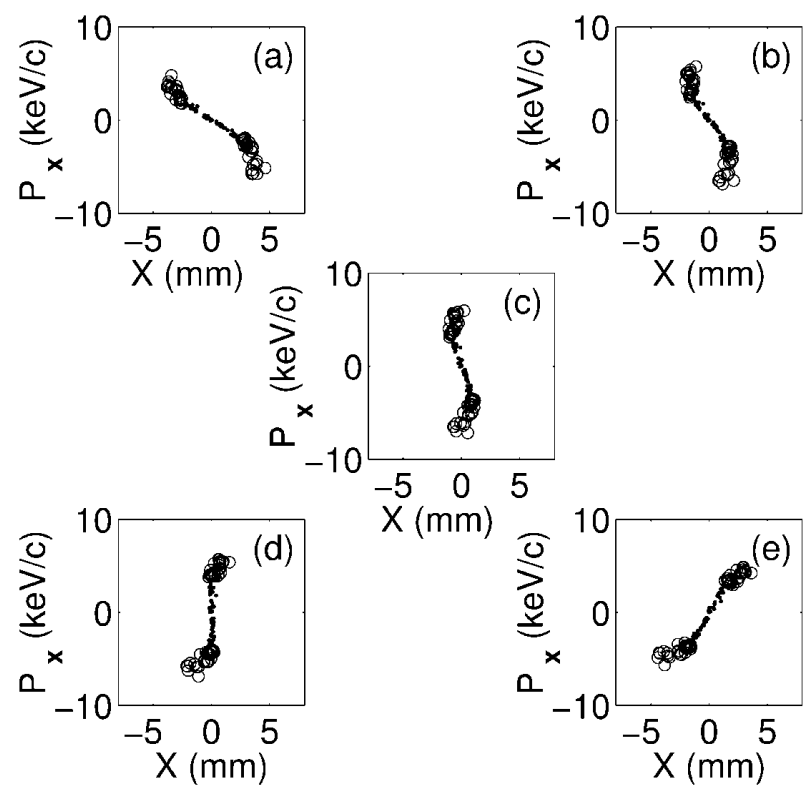

FIG. 8. Scatter plots of $p_{x}$ versus $x$ at the $s$ positions shown in Fig. 4. The high transverse momentum electrons (circles) and core electrons (points) are plotted with different symbols so that their progress may be traced near the waist. of workstations to investigate the nonlinear focusing of a $130 \mathrm{keV}, 635 \mathrm{~mA}$ plasma-focused electron beam.

\section{ACKNOWLEDGMENTS}

The author would like to thank R.E. Rand and the research, development, and engineering staff at Imatron for their support and assistance.

\section{APPENDIX A: ELLIPTICAL IMAGE ALGORITHM}

The elliptical image algorithm calculates the self-fields from the two-dimensional Fourier transform of the current density $J(x, y)$. Poisson's equation in two dimensions may be written as

$$
\left[\frac{\partial^{2}}{\partial x^{2}}+\frac{\partial^{2}}{\partial y^{2}}\right] \phi(x, y)=-\frac{\mu_{0} c}{\beta} J(x, y),
$$

We take the Fourier transform of Eq. (A1) to solve for the transform of $\phi$ in terms of the transform of $J$,

$$
\hat{\phi}\left(k_{x}, k_{y}\right)=\frac{\mu_{0} c \hat{J}\left(k_{x}, k_{y}\right)}{\beta\left(k_{x}^{2}+k_{y}^{2}\right)} .
$$

The inverse transform of Eq. (A2) yields the electrostatic potential $\phi(x, y)$, which is differentiated to obtain the selffields as in Sec. II A.

A forward FFT and inverse FFT using Eq. (A2) are performed on the grid to determine the potential $\phi(x, y)$, and 
the gradient of $\phi$ is computed numerically. Two issues must be addressed when using the FFT: (i) Eq. (A2) diverges for $k_{x}=k_{y}=0$ (the "dc" term), and (ii) the transform is performed on a finite domain, so that the potential obtained,

$$
\Phi(x, y)=\phi(x, y)+\psi(x, y),
$$

represents both the potential $\phi$ from the original beam profile and the potential $\psi$ from an infinite lattice of identical "image" profiles at positions $m_{x} L_{x} \hat{x}+m_{y} L_{y} \hat{y}$, where $m_{x}$ and $m_{y}$ are integers.

The first problem is solved by setting the dc term of the transform to zero. This changes $\phi$ by an additive constant, which vanishes upon differentiation to obtain the fields.

The second problem concerning the finite domain will require an approximation. We are interested only in $\phi(x, y)$ for positions within the beam profile. As the padded boundary region of the grid surrounding the beam profile is increased, the image contribution $\psi(x, y)$ becomes less sensitive to the detailed features of the beam profile because the image profiles are farther away. We will make the assumption that the padded boundary region is chosen sufficiently wide to allow the image profiles to be approximated as elliptically symmetric.

Nonlinear self-forces and external sextupole fields may distort the elliptical symmetry of the beam, but in many cases of practical interest the beam is approximately elliptical. The simulation of the Maxwellian beam uses $L_{x}=5 W_{x}$ and $L_{y}=5 W_{y}$, where $W_{x}$ and $W_{y}$ are the approximate dimensions of the profile along the $x$ and $y$ axes. The beam particles were binned within a restricted region at the center of the grid, so that the penalty for increasing the padding occurred only in the time required to perform the FFT on a larger grid. The validity of the assumption of elliptical symmetry for the image profiles was checked by repeating the simulation with twice the padding.

Once the assumption of elliptical symmetry is made for the image profiles, their contribution $\psi$ to the potential $\Phi$ may be subtracted using the following prescription. An elliptically symmetric model profile is created by rebinning the particle positions into elliptical rings. To determine the aspect ratio for the elliptical rings, the $n$ particles are sorted into lists of increasing $x$ position and increasing $y$ position. A particle from the $x$ list is chosen such that its $x$ position $x_{0}$ is greater than the $x$ positions for $n q$ of the remaining particles, where $q$ is a fraction chosen to be slightly less than one to reduce sensitivity to outliers. The quantity $y_{0}$ is obtained similarly from the $y$ list, and the aspect ratio is taken to be $y_{0} / x_{0}$. The model profile is next mapped onto the same FFT grid. The same FFT procedure for the model profile yields the potential $\Phi^{(m)}(x, y)$ which may similarly be decomposed into a contribution $\phi^{(m)}(x, y)$ from the original model profile and $\psi^{(m)}(x, y)$ from the image profiles. By assumption, $\psi(x, y)=\psi^{(m)}(x, y)$. The self-electric field is then given by

$$
\mathbf{E}_{s}(x, y)=\nabla \Phi^{(m)}(x, y)-\nabla \Phi(x, y)-\nabla \phi^{(m)}(x, y) .
$$

The first two gradients are computed numerically using the FFT results from the original and model profiles. The final term on the right-hand side is the electric field from the model profile; this is computed using the model profile's elliptical symmetry. Only the current enclosed by the elliptical contour through the position $(x, y)$ contributes to the field from the model profile. This region is divided into elliptical rings, and the contribution $d E_{x}^{(m)}$ and $d E_{y}^{(m)}$ from each elliptical ring is calculated analytically using the current density from the model profile. The rings are then integrated to compute the total electric field from the model profile.

The elliptical ring calculation requires the following expression for the electric field of a thin elliptical annulus with total uniform current $I$ and semiaxes $a$ (along $x$ ) and $b$ [5]:

$d E_{x}^{(m)}(x, y)=\frac{x \mu_{0} c I\left[1+\left(x^{2}+y^{2}+b^{2}-a^{2}\right) / \sqrt{\Gamma}\right]}{4 \pi \beta \sqrt{\left(a^{2}+\lambda\right)\left(b^{2}+\lambda\right)}}$,

where we have used the definitions

$\lambda=\left(x^{2}+y^{2}-a^{2}-b^{2}+\sqrt{\Gamma}\right) / 2$,

$\Gamma=\left(x^{2}+y^{2}\right)^{2}+\left(b^{2}-a^{2}\right)^{2}+2\left(x^{2}-y^{2}\right)\left(b^{2}-a^{2}\right)$.

The component $d E_{y}^{(m)}(x, y)$ may be obtained from the expression for $d E_{x}^{(m)}(x, y)$ by interchanging $x$ with $y$ and $a$ with $b$.

The wire signals from the elliptical image simulation with $1.5 \times 10^{6}$ electrons are shown in Fig. 9 at the same
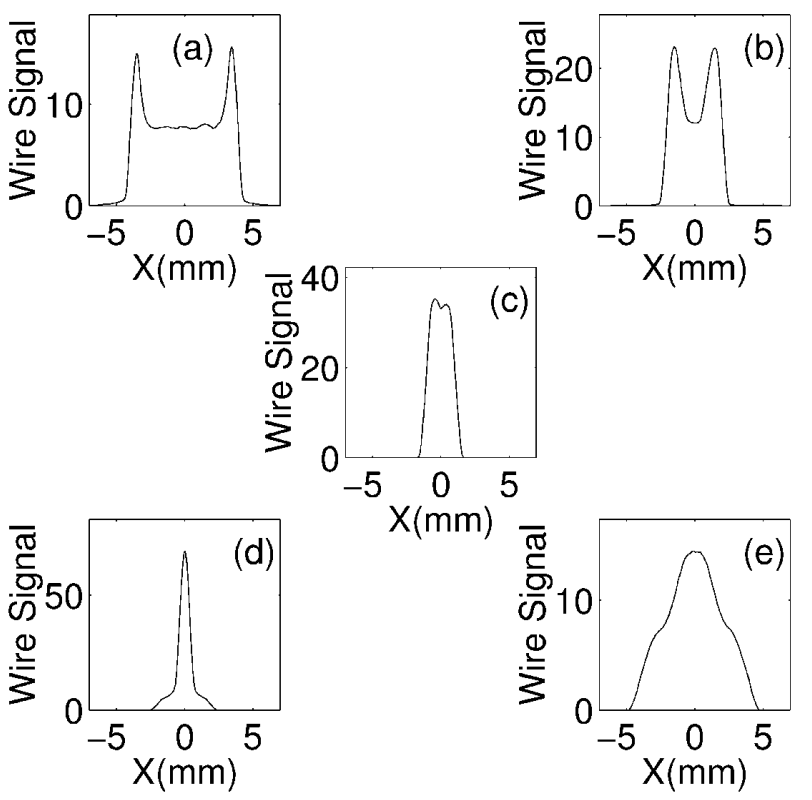

FIG. 9. Simulation of sampling wire distributions using the elliptical image algorithm to compute the self-fields. 
positions in the transport line as Fig. 5; the beam profile covered a region of roughly $50 \times 50$ elements within a grid with $256 \times 256$ elements. The simulation was also performed for the KV transport line with $4.5 \times 10^{6}$ electrons and reduced padding since the $\mathrm{KV}$ beam is elliptical; the profile covered roughly $50 \times 50$ elements and the grid contained $128 \times 128$ elements. The agreement between the simulated and theoretical beam envelopes appears identical to Fig. 2.

\section{APPENDIX B: SETTING THE KERNEL TO ZERO AT THE ORIGIN IN HOCKNEY'S ALGORITHM}

The current distribution $J(x, y)$ is approximated as an ensemble of current filaments located at the centers $\left(x_{k}, y_{k}\right)$ of each bin in the grid, and the kernel at the origin represents the response from a single filament at the position of the filament. Denote the total current for the filament in bin $k$ as $I_{k}$. This current is taken to be nonzero (although arbitrarily small outside the beam) and uniformly distributed over an infinitesimal disk of radius $\epsilon_{k}$. The potential at a distance $r<\epsilon_{k}$ from the center of the disk is given by

$$
\phi\left(r<\epsilon_{k}\right)=-\frac{\mu_{0} c}{4 \pi \beta}\left[\frac{I_{k} r^{2}}{\epsilon_{k}^{2}}+I_{k}\left(2 \log \epsilon_{k}-1\right)\right],
$$

where $\phi(0)$ is determined by requiring consistency with Eq. (2.3) at $r=\epsilon_{k}$. At $r=0$ the integral in Eq. (2.2) contributes $\phi(0)$, which depends upon $\epsilon_{k}$. We require the electric field to be independent of our choice for $\epsilon_{k}$. Since $\epsilon_{k}$ is infinitesimal, we are free to adjust it for each bin so that quantity $I_{k}\left(2 \log \epsilon_{k}-1\right)$ is constant. Then the constant $\phi(0)$ vanishes upon differentiation to obtain the electric field; hence, the same result is obtained by taking this constant to be zero in our definition of the kernel.

Setting the kernel to zero at the origin is a slight modification of the algorithm in Ref. [3], which assigns the value one to the kernel at the origin, thereby adding the small term $I_{k}$ to the potential $\phi\left(x_{k}, y_{k}\right)$. The simulations in this report were repeated using one for the kernel at the origin with negligible changes in the results.

[1] R. E. Rand, M. C. Lampel, and D. Y. Wang, J. Appl. Phys. 62, 1639 (1987).

[2] J.D. Lawson, The Physics of Charged Particle Beams (Clarendon, Oxford, 1977), pp. 134, 190, 195, 196, 199, and 221.

[3] R. W. Hockney and J. W. Eastwood, Computer Simulation Using Particles (Adam Hilger, New York, 1988), pp. 94, 211-214.

[4] R.E. Rand and J. L. Couch, U.S. Patent No. 4,631,741 (1986).

[5] S. Chandrasekhar, Ellipsoidal Figures of Equilibrium (Dover, New York, 1969). Equation (A5) was derived from Eq. 56, p. 46. 